\title{
Bloodstream infection caused by Yersinia enterocolitica in a host with ankylosing spondylitis: a case report and literature review
}

\author{
Yang-Xi Liu, Han Zhong, Ke-Jia Le, Min Cui \\ Department of Pharmacy, Renji Hospital, School of Medicine, Shanghai Jiaotong University, Shanghai, China \\ Correspondence to: Min Cui. Renji Hospital, School of Medicine, Shanghai Jiaotong University, Shanghai 200127, China. Email: cuimin@renji.com.
}

\begin{abstract}
The extraintestinal infections caused by Yersinia enterocolitica are very rare, especially in the form of spontaneous bloodstream infection at people without history of blood transfusion. Their clinical symptoms and treatments are still not very clear for now. Here, we report a case of spontaneous bloodstream infection caused by $Y$. enterocolitica in a 56-year-old Chinese male. The patient presented to outpatient with fever for 1 week, he was diagnosed ankylosing spondylitis for 10 years, and suffered from the pain in his neck, lumbosacral region and limbs constantly. After 4 days of outpatient treatment, there was no sign of improvement so he admitted to inpatient department. Ceftriaxone and metronidazole were initiated in the previous 3 days, the temperature did not drop (highest temperature is $38.3^{\circ} \mathrm{C}$ ) and the limb joint pain was aggravated. On day 4, antibiotic therapy was changed to moxifloxacin as the growth of $Y$. enterocolitica showed in blood culture, then changed to amikacin and piperacillin/tazobactam according to culture susceptibility. The patient received a total of 24 days antibacterial treatment before discharge, his body temperature returned to normal, but he remains continuous pain in lumbosacral region and limbs after negative blood culture, which was considered to be caused by AS. Gastrointestinal symptoms such as vomiting, diarrhea and abdominal pain were not reported during the hospitalization, which usually appears in patients with Yersinia enterocolitica infection. We reviewed 12 septicemia cases without the history of blood transfusion from the literature. Not all hosts were under a low immunity or have a clear history of exposure. Clinical symptoms and antibiotic agents were also different from case to case. Physicians should consider the rare diagnosis of $Y$. enterocolitica infection in patients without clear history of exposure and typical symptoms. And distinguish between pain caused by AS and aseptic arthritis caused by Y. enterocolitica.
\end{abstract}

Keywords: Yersinia enterocolitica; bloodstream infection; antibacterial treatment; clinical symptoms; case report

Submitted Jan 19, 2020. Accepted for publication May 21, 2020.

doi: $10.21037 / \mathrm{apm}-20-256$

View this article at: http://dx.doi.org/10.21037/apm-20-256

\section{Introduction}

Genus Yersinia, the Gram-negative coccobacilli has 11 species, three of which can cause disease in humans, including Yersinia pestis, Yersinia pseudotuberculosis and Yersinia enterocolitica (1). Y. enterocolitica mainly cause gastroenteritis through contaminated food or water, and it is also considered as a common factor of chronic aseptic arthritis. Extraintestinal complications caused by $Y$. enterocolitica are highly unusual, septicemia can occur after acute yersiniosis or secondary complications in immunocompromised hosts, and other reported septicemia with $Y$. enterocolitica are related to blood transfusion (2).

However, bloodstream infection without gastrointestinal or other systemic infections are extremely rare and the treatment is still not very clear. We report a case of bloodstream infection caused by $Y$. enterocolitica in a patient with 10 -year ankylosing spondylitis, which the joint symptoms are very similar to aseptic arthritis caused by $Y$. enterocolitica. After a combined antimicrobial therapy according to antimicrobial susceptibility for 24 days, the patient was recovered and there is no sign of recurrence. We discussed this case and present the following case in accordance with the CARE Reporting Checklist, meanwhile 
Table 1 Antimicrobial susceptibility of Yersinia enterocolitica in our case

\begin{tabular}{lcc}
\hline Antibiotics & MIC $(\mu \mathrm{g} / \mathrm{mL})$ & Category \\
\hline Amikacin & 16 & $\mathrm{~S}$ \\
Gentamycin & $\geq 16$ & $\mathrm{R}$ \\
Ampicillin/sulbactam & $32 / 16$ & $\mathrm{R}$ \\
Piperacillin/tazobactam & $\leq 2 / 4$ & $\mathrm{~S}$ \\
Cefuroxime & $\geq 32$ & $\mathrm{R}$ \\
Ceftazidime & 16 & $\mathrm{R}$ \\
Cefotaxime & 8 & $\mathrm{R}$ \\
Cefepime & 1 & $\mathrm{~S}$ \\
Ciprofloxacin & 1 & $\mathrm{~S}$ \\
Levofloxacin & 1 & $\mathrm{~S}$ \\
Imipenem & 1 & $\mathrm{~S}$ \\
Meropenem & $\leq 0.06$ & $\mathrm{~S}$ \\
Minocycline & 4 & $\mathrm{~S}$ \\
Sulfamethoxazole/trimethoprim & $\leq 2 / 38$ & $\mathrm{~S}$ \\
\hline
\end{tabular}

MIC, minimum inhibitory concentration; $S$, sensitive; $R$, resistance.

we reviewed other 12 cases with reference in this article.

\section{Case presentation}

A 56-year-old Chinese male with a history of 10-year AS, presented to outpatient clinic with fever, and he also suffered from the pain in his neck, lumbosacral region and limbs constantly. The patient did not report experiencing any gastrointestinal symptoms and the highest temperature is $38.3^{\circ} \mathrm{C}$. He did not take medicine regularly for AS, only in the onset of symptoms using NSAIDs such as loxoprofen to control the pain. He also denied any recent travel, blood transfusion, unclean food and animal contact. After 4 days of symptomatic treatment the temperature did not drop and the pain at limb joint worsened so he admitted to hospitalization. On admission, his temperature is $38^{\circ} \mathrm{C}$, the other vital signs were stable. Physical examination revealed limited activities of lumbar and cervical vertebrae. Laboratory data on admission showed as follows: neutrophile granulocyte $80.7 \%$ (reference, 50-70\%); CRP $115.29 \mathrm{mg} / \mathrm{L}$ (reference, 0-8 mg/L); ESR $20 \mathrm{~mm} / \mathrm{h}$ (reference, $0-15 \mathrm{~mm} / \mathrm{h}$ ); PCT $0.191 \mathrm{ng} / \mathrm{mL}$ (reference, $<0.05 \mathrm{ng} / \mathrm{mL}$ ), other laboratory parameters are within normal limits. X-ray and CT showed double sacroiliac joint fusion, in line with the changes after ankylosing spondylitis, and no indication of infection.

Blood culture was taken before ceftriaxone ( $2 \mathrm{~g}$ once a day) and metronidazole ( $0.5 \mathrm{~g} 3$ times daily) combination therapy on the day of admission (hospital day 1), NASIDs were initiated to control the pain. On hospital day 4 the blood culture showed $Y$. enterocolitica grows and it is sensitive to amikacin, minocycline, carbapenems and quinolones (Table 1). Antibiotics changed to moxifloxacin ( $0.4 \mathrm{~g}$ once a day) according to the sensitivity of culture results. The pain had slightly relieved but his body temperature did not return to normal after the treatment with moxifloxacin for two days, so he was transferred to a superior hospital on the hospital day 6. Blood culture was taken on the first day of superior hospital (hospital day 7) which yielded negative results, laboratory data of routine blood test was normal, only CRP was $200 \mathrm{mg} / \mathrm{L}$ (reference, $0-8 \mathrm{mg} / \mathrm{L}$ ) and the highest temperature was $38.2^{\circ} \mathrm{C}$. Amikacin $(0.6 \mathrm{~g}$ once a day) and piperacillin/tazobactam ( $4.5 \mathrm{~g} 3$ times daily) were given until discharge (total 18 days), the patient's temperature returned to normal on the fifth day of superior hospital (hospital day 11). Multiple blood cultures during hospitalization were negative. The patient received a total of 24 days of combined antibiotic therapy without side effect until discharge. Pain in the lumbosacral and cervical vertebrae was lessened but did not cure, and no redness or swelling was found in the joints. Until the time of writing the patient has shown no recurrence of fever. The details of historical and current treatment were showed on Table 2. Written informed consent was obtained from the patient for publication of this case report and any accompanying images.

\section{Literature review}

We reviewed past case reports of bloodstream infections, including septicemia or sepsis, caused by $Y$. enterocolitica through PubMed (http://www.ncbi.nlm.nih.gov/pubmed; accessed November 1, 2019), the reports of infections by blood transfusion were excluded. The search use terms "Yersinia enterocolitica" combined with "septicemia" or "septic", "bloodstream infection", "BSI", "blood poisoning", "sepsis" from January 1, 1969 to November 1, 2019. 12 articles (12 cases) fit this definition (Table 3).

\section{Discussion}

Yersinia enterocolitica is a Gram-negative, facultatively 
Table 2 Treatment and timeline of the present case

\begin{tabular}{lll}
\hline Days of admission & Symptoms and diagnosis & Antimicrobial therapy \\
\hline Day 1-3 & Fever, pain in neck, lumbosacral region and limbs & Ceftriaxone (2 g once daily) and metronidazole (0.5 g 3/d) \\
Day 4 & Bacteremia of Y. enterocolitica & Moxifloxacin (0.4 g once daily) \\
Day 5-6 & $\begin{array}{l}\text { Fever, pain in lumbosacral and cervical vertebrae had } \\
\text { slightly relieved }\end{array}$ & \\
Day 7-11 & Fever, intermittent pain & Amikacin $(0.6 \mathrm{~g}$ once daily) and piperacillin/ \\
Day11-24 & Normal temperature, Intermittent pain in neck, & tazobactam (4.5 g 3/d) \\
& lumbosacral region and limbs but lessened & \\
\hline
\end{tabular}

3/d, three times daily.

anaerobic coccobacillus, it is also considered as an enteropathogen which usually causing relatively mild disease and septicemia caused by $Y$. enterocolitica is very rare (15). Even with bloodstream infection, most hosts have a history of blood transfusion that the red blood cell may be contaminated by $Y$. enterocolitica (1). We reported a case of primary bloodstream infection caused by $Y$. enterocolitica, which is very rare in a host without blood transfusion.

The usual route of acquisition of $Y$. enterocolitica is through contacting with contaminated food (including raw pork or unpasteurized milk) or infected animal. As all species of genus Yersinia are motile at $22-30{ }^{\circ} \mathrm{C}$ but not at $37^{\circ} \mathrm{C}$ expect $\mathrm{Y}$. pestis, and $Y$. enterocolitica withstands freezing very well and may survive for prolonged periods in frozen food. Infections with $Y$. enterocolitica are more common at cold regions or colder seasons (16-19), and in our case, it was winter when the patient developed fever and body pain. The patient denied any blood transfusion, recent travel and animal contact. As the same food was consumed by his family but they didn't show any similar symptoms, we also excluded the infection by unclean food. As we known, Y. enterocolitis is also a common cause of chronic aseptic arthritis, especially in in patients with compromised immune function. The pathogenesis is likely due to an immune response that Yersinia antigens cross-reacts with host antigens, which the host has HLA-B27 positivity making them susceptible to arthritis (20). The patient has a history of 10-year AS, he suffered from the pain caused by AS constantly. We conclude the pain in his neck, lumbosacral region and limbs were mostly caused by AS, because X-ray and CT both showed no indication of infection and in line with joint changes of AS. As the patient's immune status was not clear, we inferred the pathogen was $Y$. enterocolitica have directly entered into the blood through an infected tissue, which means the patient is under an asymptomatic carrier state. Our review of the literature (Table 3) demonstrate in some cases, septicemia can occur without exposure to the source of the contamination and most of those infected are in a state of low immunity.

Septicemia with $Y$. enterocolitica pathogenesis is colonization of the intestinal tract, especially the distal small intestine and proximal colon, and penetrate the mucus barrier, causing an inflammatory response that triggers gastrointestinal symptoms such as diarrhoea, abdominal pain, nausea and vomiting (2). In our case, the patient showed fever and pain in his neck, lumbosacral region and limbs, but no gastrointestinal symptoms. We reviewed the 12 similar cases (Table 3), although clinical presentation of bloodstream infection caused by $Y$. enterocolitica can range from a normal temperature state to fever, multiple organ abscesses, septic arthritis and osteomyelitis to disseminated disease, but abdominal discomfort is common and cases without gastrointestinal symptoms are extremely rare.

For the great majority of gastrointestinal infections caused by $Y$. enterocolitica are self-limiting and do not merit antimicrobial therapy in an immunocompetent host. However, antibiotics are warranted for extraintestinal infections including septicemia, in which the mortality can be as high as $50 \%$ (2). Although the septicemia with $Y$. enterocolitica can lead to complications including liver abscess, arthritis and other symptoms, timely administration of effective antibiotics seems to improve the prognosis. According to our literature review, most cases were recovered after proper antibiotics treatment.

The initial recommendations for antibiotics by WHO including tetracycline, gentamicin and TMP/ SMZ. Recently, third generation cephalosporins and fluoroquinolones showed excellent in vitro activity to $Y$. enterocolitica, which have been considered as better alternatives (2). In our case, blood culture showed 


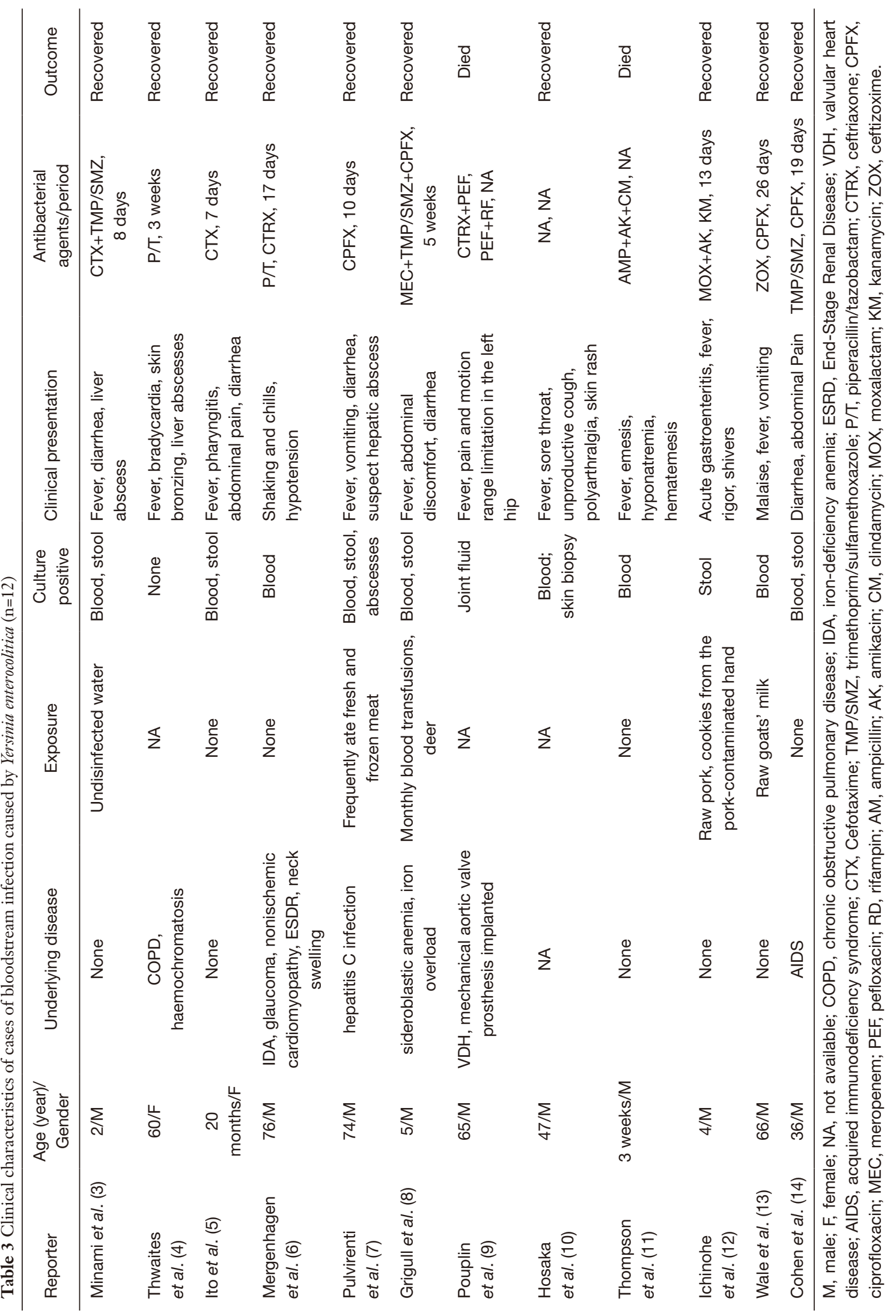


$Y$. enterocolitica was resistance to gentamicin, some third generation cephalosporins, but sensitive to fluoroquinolones, carbapenems, amikacin and piperacillin/ tazobactam. Ceftriaxone and metronidazole were given for 3 days, then changed to moxifloxacin according to the sensitivity of culture results. Although the patient's temperature did not return to normal, the blood culture and laboratory data of routine blood test were normal after treatment. As soon as combination therapy changed to amikacin and piperacillin/tazobactam, clear improvements were achieved within a few days. This case was successfully treated with the combination of appropriate antibiotics for 24 days. Most past cases have also improved after implementing effective antibiotics including third generation cephalosporins, fluoroquinolones and aminoglycosides. No recurrences were identified and the longest therapy lasted 5 weeks.

This case has limitations and must be interpreted with care. The infections caused by $Y$. enterocolitica are extremely rare and differences exist in individuals, so the regimen and full course of antibiotic treatment are still uncertain. What antimicrobial agent to select and when to stop are usually in accordance with blood culture. In our case, moxifloxacin is sensitive to culture $Y$. enterocolitica but the therapeutic effect was not significant. Even after the patient's temperature and blood culture returned to normal, the patient still continued antimicrobial agents due to lack of literature support. The limitations restrict the broader applicability of the treatment in this case.

\section{Conclusions}

Septicemia with $Y$. enterocolitica is a rare event, exact incidence can only be estimated by recording of all occurrences at a multinational scale systematically and antimicrobial regimens vary from country to country. Clinical symptoms and route of acquisition also vary in different patient. This case involved spontaneous bloodstream infection caused by $Y$. enterocolitica in an AS patient without history of source exposure and gastrointestinal symptoms did not appear during hospitalization. He was successfully treated with a combined antibiotic therapy for 28 days. The pain caused by AS may easily confused with arthritis caused by $Y$. enterocolitica and physician should be alert to distinguish them. And physician should consider rare diagnosis in patients without typical symptoms.

\section{Acknowledgements}

Funding: Shanghai youth pharmacist training program (Clinical Pharmacy, 2017), Research Funds of Shanghai Health and Family Planning commission (20184Y0022), Cultivation fund of clinical research of Renji Hospital (PY2018-III-06).

\section{Footnote}

Conflicts of Interest: All authors have completed the ICMJE uniform disclosure form (available at http://dx.doi. org/10.21037/apm-20-256). The authors have no conflicts of interest to declare.

Etbical Statement: The authors are accountable for all aspects of the work in ensuring that questions related to the accuracy or integrity of any part of the work are appropriately investigated and resolved. Written informed consent was obtained from the patient for publication of this case report and any accompanying images.

Open Access Statement: This is an Open Access article distributed in accordance with the Creative Commons Attribution-NonCommercial-NoDerivs 4.0 International License (CC BY-NC-ND 4.0), which permits the noncommercial replication and distribution of the article with the strict proviso that no changes or edits are made and the original work is properly cited (including links to both the formal publication through the relevant DOI and the license). See: https://creativecommons.org/licenses/by-nc-nd/4.0/.

\section{References}

1. Bottone EJ. Yersinia enterocolitica: the charisma continues. Clin Microbiol Rev 1997;10:257-76.

2. Fabrega A, Vila J. Yersinia enterocolitica: pathogenesis, virulence and antimicrobial resistance. Enferm Infecc Microbiol Clin 2012;30:24-32.

3. Minami K, Yasuda R, Terakawa R, et al. Four Sporadic Pediatric Cases of Yersinia enterocolitica O:8 Infection in a Rural Area of Japan. Jpn J Infect Dis 2017;70:192-4.

4. Thwaites PA, Woods ML. Sepsis and siderosis, Yersinia enterocolitica and hereditary haemochromatosis. BMJ Case Rep 2017;2017.

5. Ito T, Suzuki T, Kawase J, et al. Yersinia enterocolitica bacteremia and enterocolitis in a previously healthy 20-month-old girl. J Infect Chemother 2012;18:756-9. 
6. Mergenhagen KA, Telesz AL. Yersinia Enterocolitica Bacteremia in a Chronic, Mildly Iron-Overloaded Dialysis Patient. Ann Pharmacother 2011;45:e14.

7. Pulvirenti D, Aikaterini T, Neri S. Septicemia, hepatic abscess, and encephalitis due to Yersinia enterocolitica. J Clin Gastroenterol 2007;41:333-4.

8. Grigull L, Linderkamp C, Sander A, et al. Multiple spleen and liver abscesses due to Yersinia enterocolitica septicemia in a child with congenital sideroblastic anemia. J Pediatr Hematol Oncol 2005;27:624-6.

9. Pouplin S, Daragon A, Cambon-Michot C, et al. Septic arthritis of the hip caused by Yersinia enterocolitica: a case report. Joint Bone Spine 2002;69:604-6.

10. Hosaka $S$, Uchiyama $M$, Ishikawa $M$, et al. Yersinia enterocolitica serotype O:8 septicemia in an otherwise healthy adult: analysis of chromosome DNA pattern by pulsed-field gel electrophoresis. J Clin Microbiol 1997;35:3346-7.

11. Thompson EC. Yersinia enterocolitica sepsis in a 3-weekold child. J Natl Med Assoc 1994;86:783-5.

12. Ichinohe $H$, Yoshioka $M$, Fukushima $H$, et al. First isolation of Yersinia enterocolitica serotype O:8 in Japan. J Clin Microbiol 1991;29:846-7.

13. Wale MC, Liddicoat AJ, Pether JV. Yersinia enterocolitica

Cite this article as: Liu YX, Zhong H, Le KJ, Cui M. Bloodstream infection caused by Yersinia enterocolitica in a host with ankylosing spondylitis: a case report and literature review. Ann Palliat Med 2021;10(5):5780-5785. doi: 10.21037/apm-20-256 biotype 2 serotype $\mathrm{O} 9$ septicaemia in a previously fit man, raw goats' milk having been the apparent vehicle of infection: a cautionary tale. J Infect 1991;23:69-72.

14. Cohen JI, Rodday P. Yersinia enterocolitica bacteremia in a patient with the acquired immunodeficiency syndrome. Am J Med 1989;86:254-5.

15. Guinet F, Carniel E, Leclercq A. Transfusiontransmitted Yersinia enterocolitica sepsis. Clin Infect Dis 2011;53:583-91.

16. Arun R, Kasbekar AV, Mehdian SM. Spontaneous kyphotic collapse followed by autostabilisation secondary to cervical osteomyelitis in an intravenous drug abuser. Acta Orthop Belg 2007;73:807-11.

17. Berner R, Kist M, Sauer M. Encephalopathy associated with Yersinia enterocolitica O:3. Lancet 1998;351:418.

18. Leclercq A, Martin L, Vergnes ML, et al. Fatal Yersinia enterocolitica biotype 4 serovar O:3 sepsis after red blood cell transfusion. Transfusion 2005;45:814-8.

19. von Eckardstein K, Spuler A, Brauer C, et al. Spontaneous cervical osteomyelitis due to Yersinia enterocolitica in a non-immunocompromised host. Eur J Clin Microbiol Infect Dis 2004;23:66-8.

20. Aziz M, Waheed A. Yersinia Enterocolitica. StatPearls. Treasure Island (FL) 2019. 